\title{
Compatibility of a Colloidal Oatmeal Containing Body Wash for Pediatric Subjects with Atopic Dermatitis
}

\section{Arrowitz $C E^{a}$, Jiang $L^{b}$, Hino $P D^{b}$, Weber TMa}

aBeiersdorf Inc., Wilton, CT, USA. ' ${ }^{\text {T}}$ Thomas J. Stephens \& Associates, Inc., Richardson, TX, USA.

\section{ABSTRACT}

Background: Cleansing is an important part of regular hygiene, especially for patients with atopic dermatitis (AD) due to their susceptibility to skin infections. However, for these patients, regular cleansing with soaps and detergents can irritate their sensitive skin and exacerbate flares. Current AAD guidelines for the management of $A D$ recommend use of non-soap cleansers that are low-pH, hypoallergenic, and fragrance-free. The following clinical trial tested low-phiall formulated Mild Cream Body Wash (MCBW) containing $2 \%$ colloida atmeal (skin protectant), mild surfactants, Ceramid NP, and components of oatmeal (skin protectant), mild surfactants, Ceramide NP, and components of the natural moisturizing factor on children with AD. The MCBW formula was further optimized for this population by its low $\mathrm{pH}$, and fragrance-, dye-, and soap-free properties.

Aim: This clinical trial was conducted to test the tolerability of a MCBW when used for regular bathing (once daily) by children and young adults with clinically confirmed AD.

Method: Study duration was 2 weeks with tolerability evaluations (clinical grading) and self-assessment questionnaires (completed by a parent/guardian when appropriate) conducted at baseline, Week 1, and Week 2 clinical visits. 35 male and female children, ages 2 months to 18 years old were included in the following three age bands: 2 months through 24 months n=10, 25 months through 12 years $n=14,13$ years to 18 years $n=11$. Subjects were instructed to use only the MCBW for their bathing routines.

Results: The MCBW was well tolerated by all subjects. Statistically significant $(p<0.001)$ reductions in clinically graded erythema, dryness, and itching were observed at Weeks 1 and 2 compared to baseline (pre-cleanser use). Additionally, significantly fewer subjects reported itching $(p<0.05)$ and dryness $(p<0.001)$ after bathing at Weeks 1 and 2 compared to baseline. After 1 week of use, $91.4 \%$ of parents/guardians found that the cleanser was gentle on their child's eczematic/eczema-prone skin, and $88.6 \%$ agreed the cleanser was gentle enough for everyday use.

Conclusion: MCBW was very well tolerated by pediatric subjects with clinically confirmed AD. The results demonstrate that the skin protectant body wash is well-suited for everyday use by subjects with $A D$, helping to reduce the irritation caused by regular bathing.

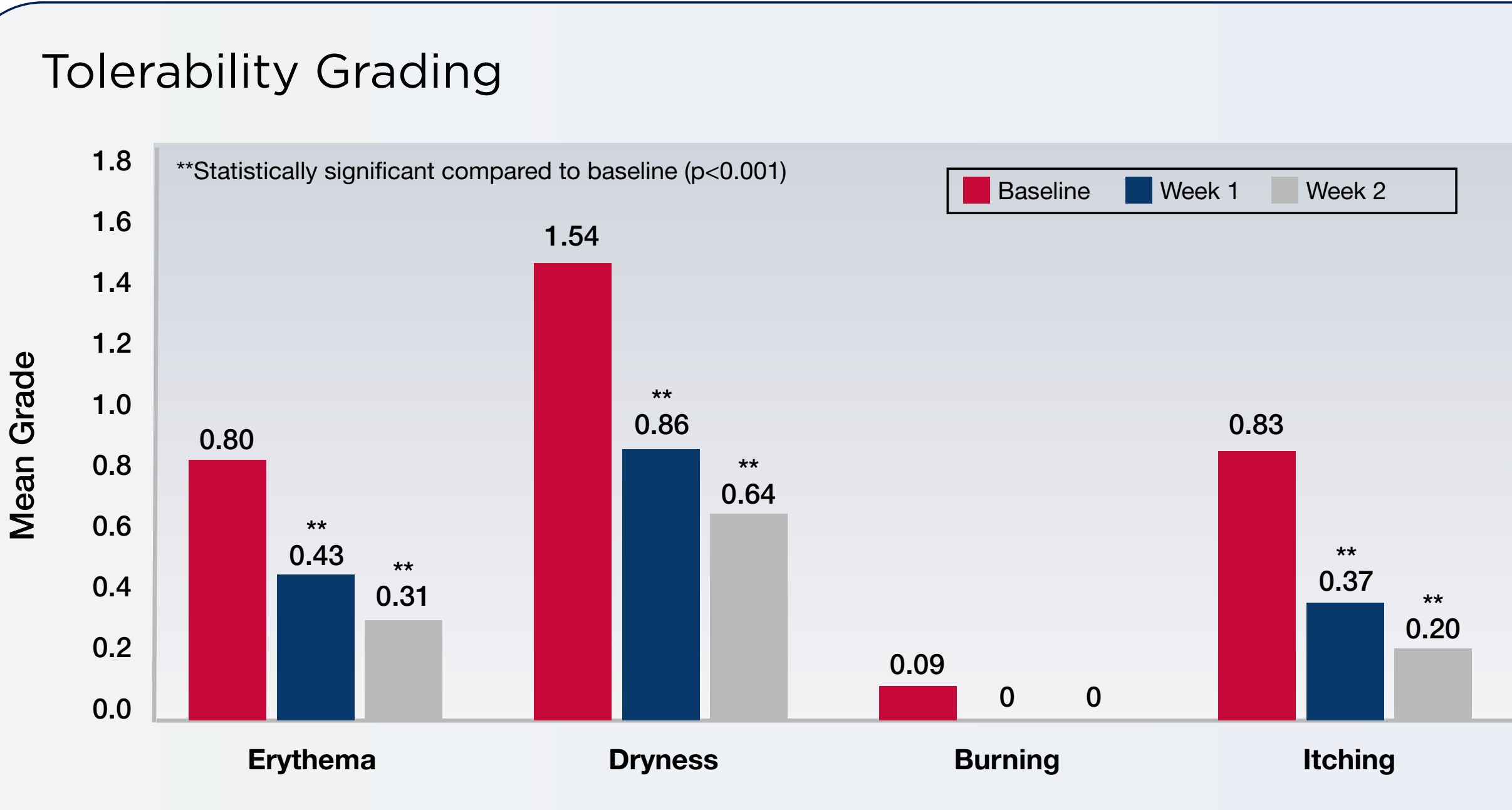

Figure 1. Tolerability Grading: Mean Scores. Tolerability evaluations for erythema, dryness, burning, and itching were conducted at baseline, Week 1 , and Week 2 using a 0 to 3 scale (0=none to $3=$ severere). Subjects $(N=35)$ bathed daily
utilizing the Mild Cream Body Wash as part of their bathing routine for two weeks.

Perception of Tolerability (Week 1)

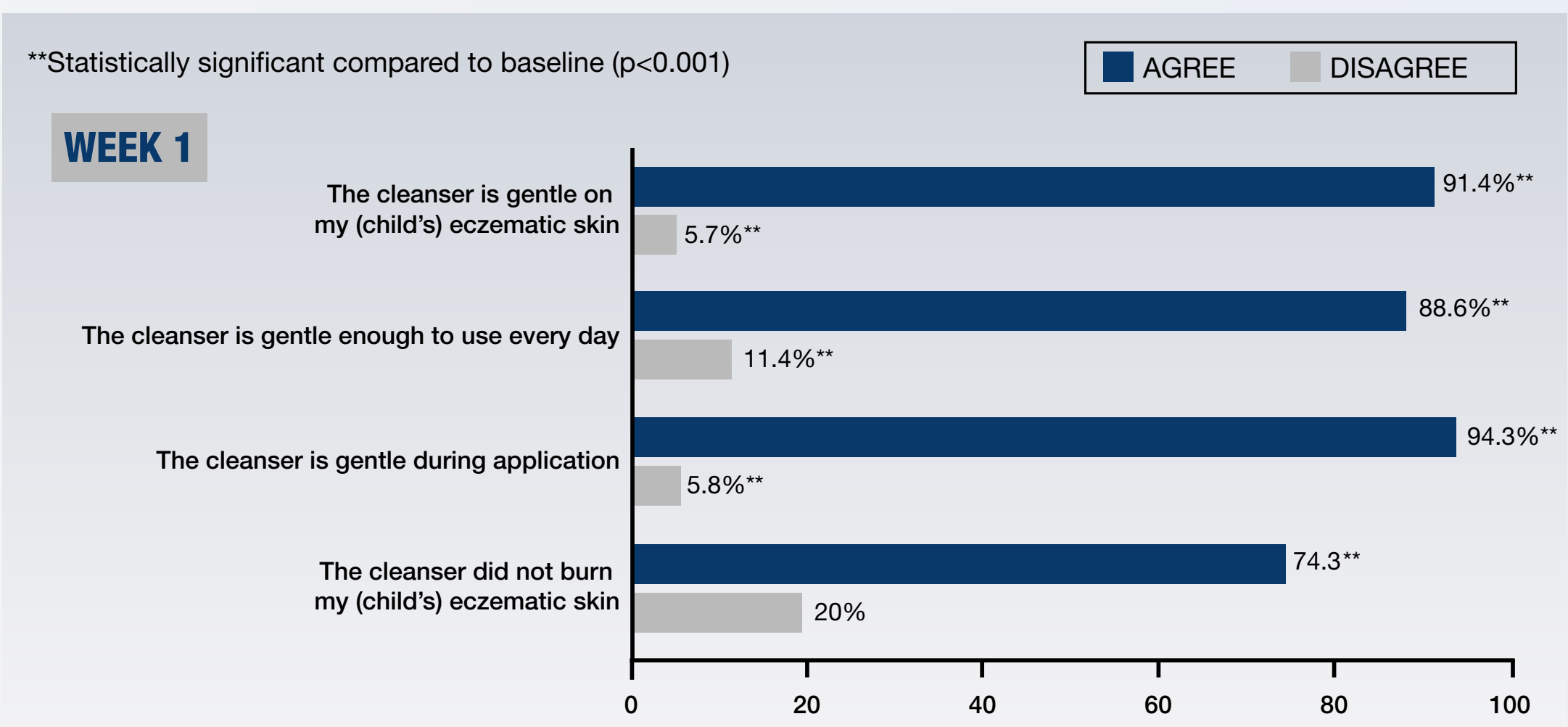

Perception of Tolerability (Week 2)

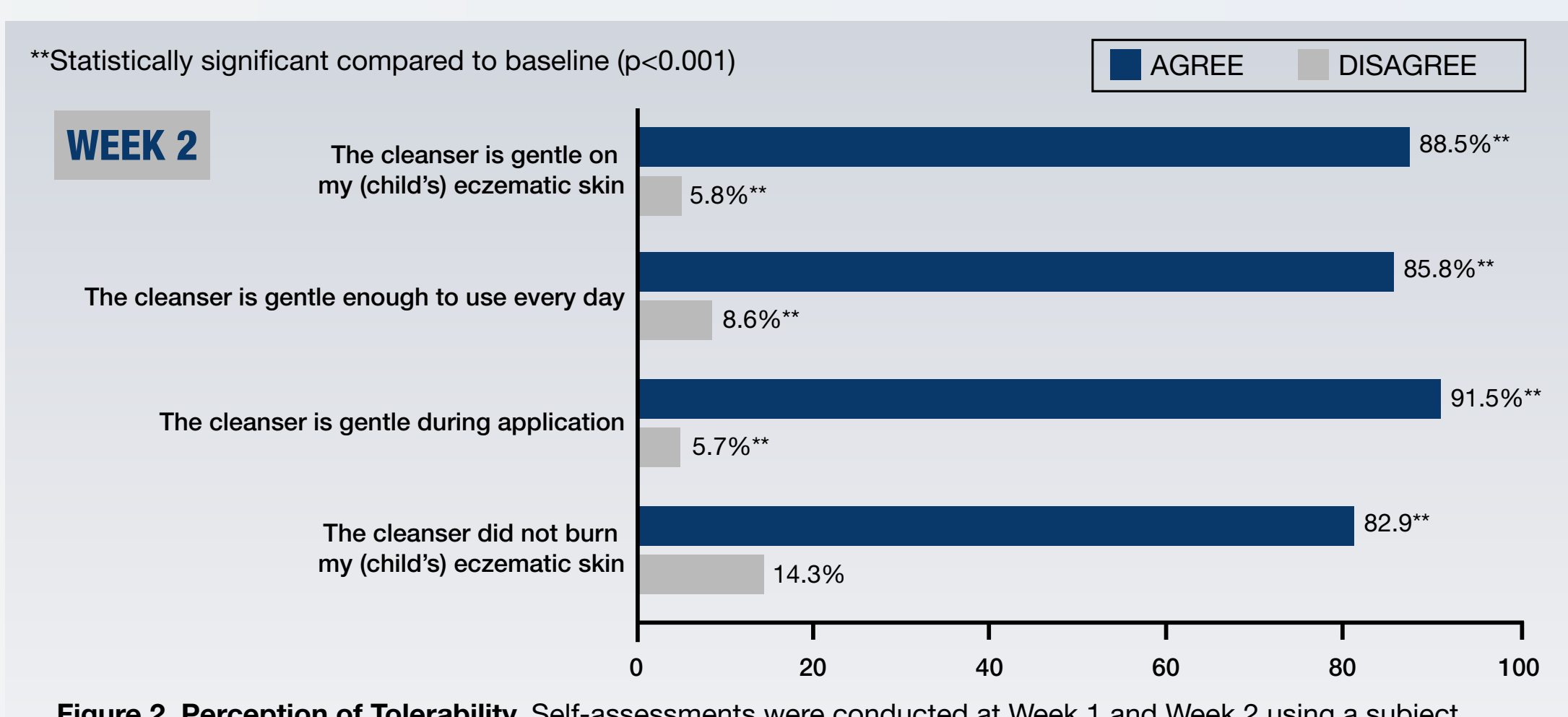

Figure 2. Percerion of Tolerability. Self-assessments were conducted at Week 1 and Week 2 using a subject
My (child's) skin is irritated after bathing

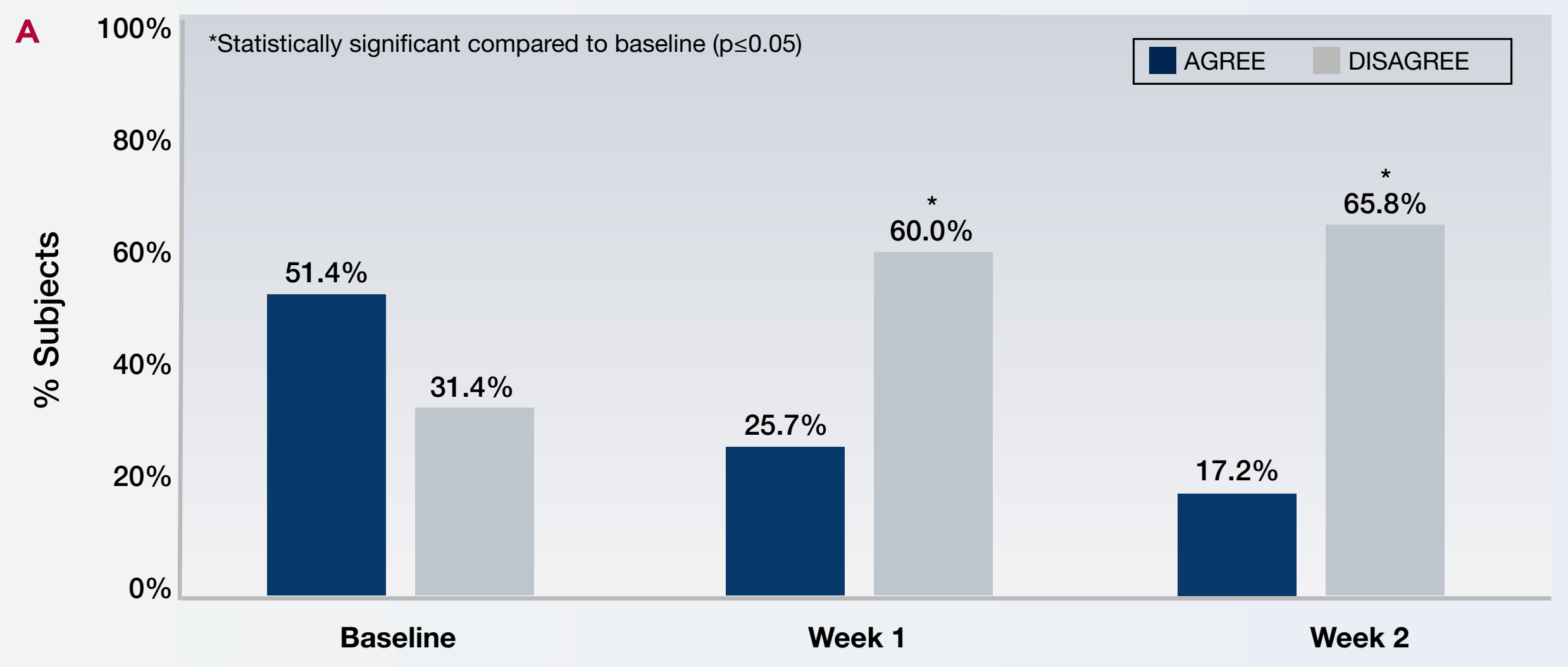

My (child's) skin itches after bathing

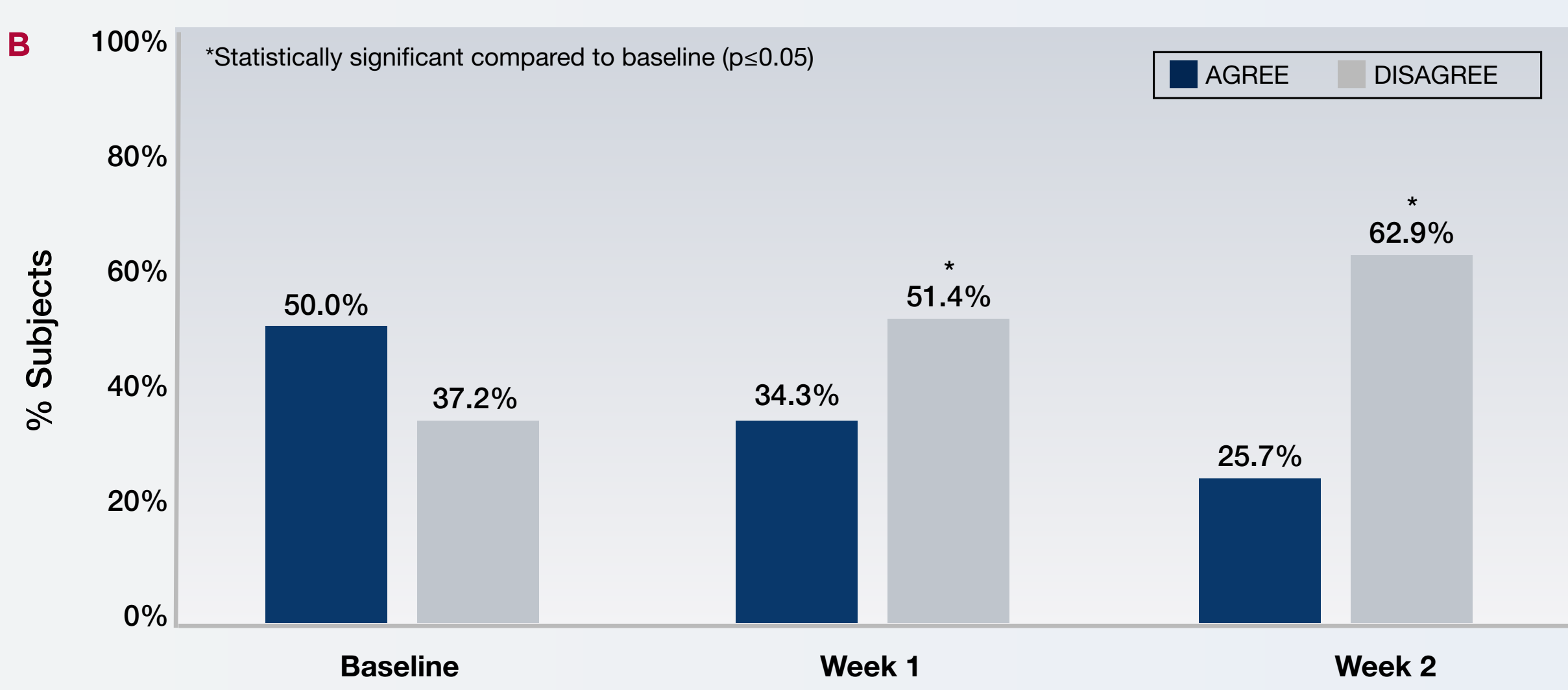

My (child's) skin feels dry after bathing

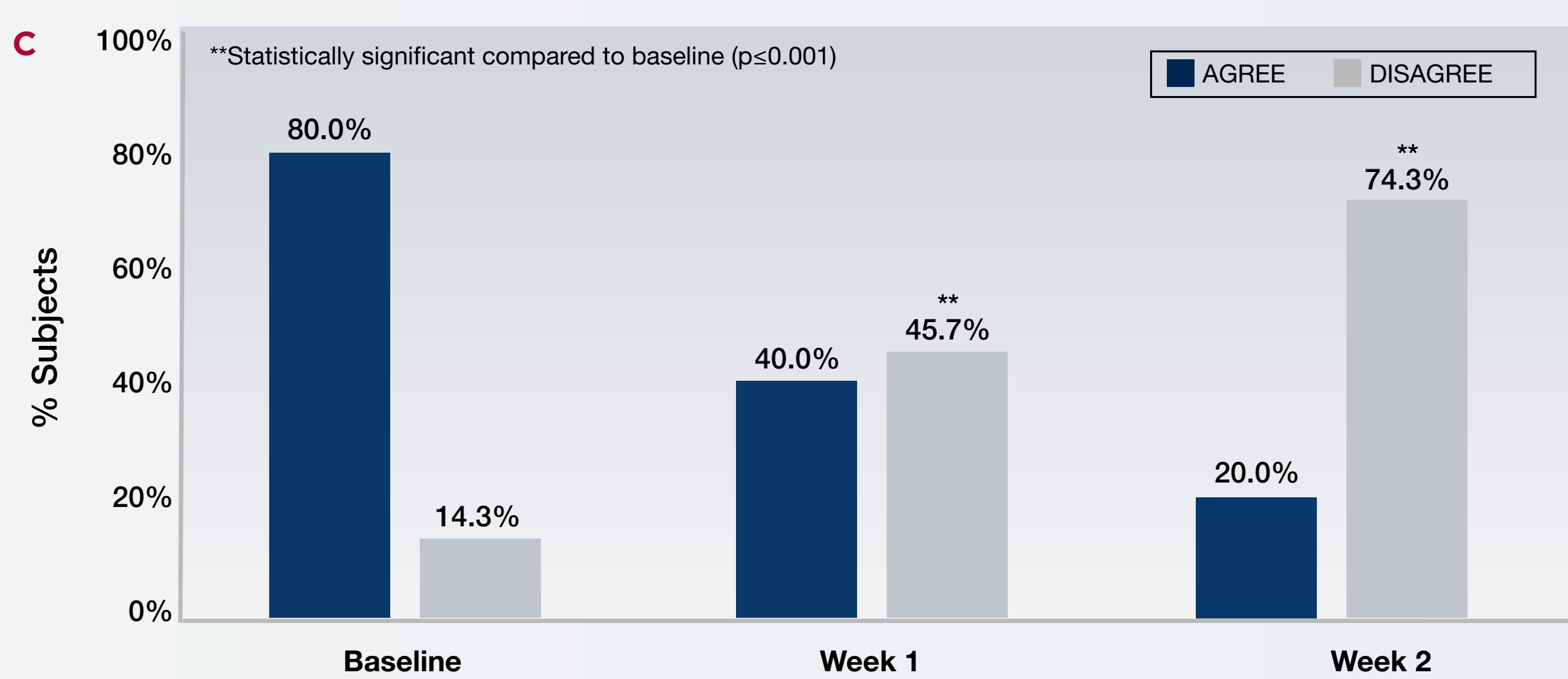

My (child's) skin appears red after bathing

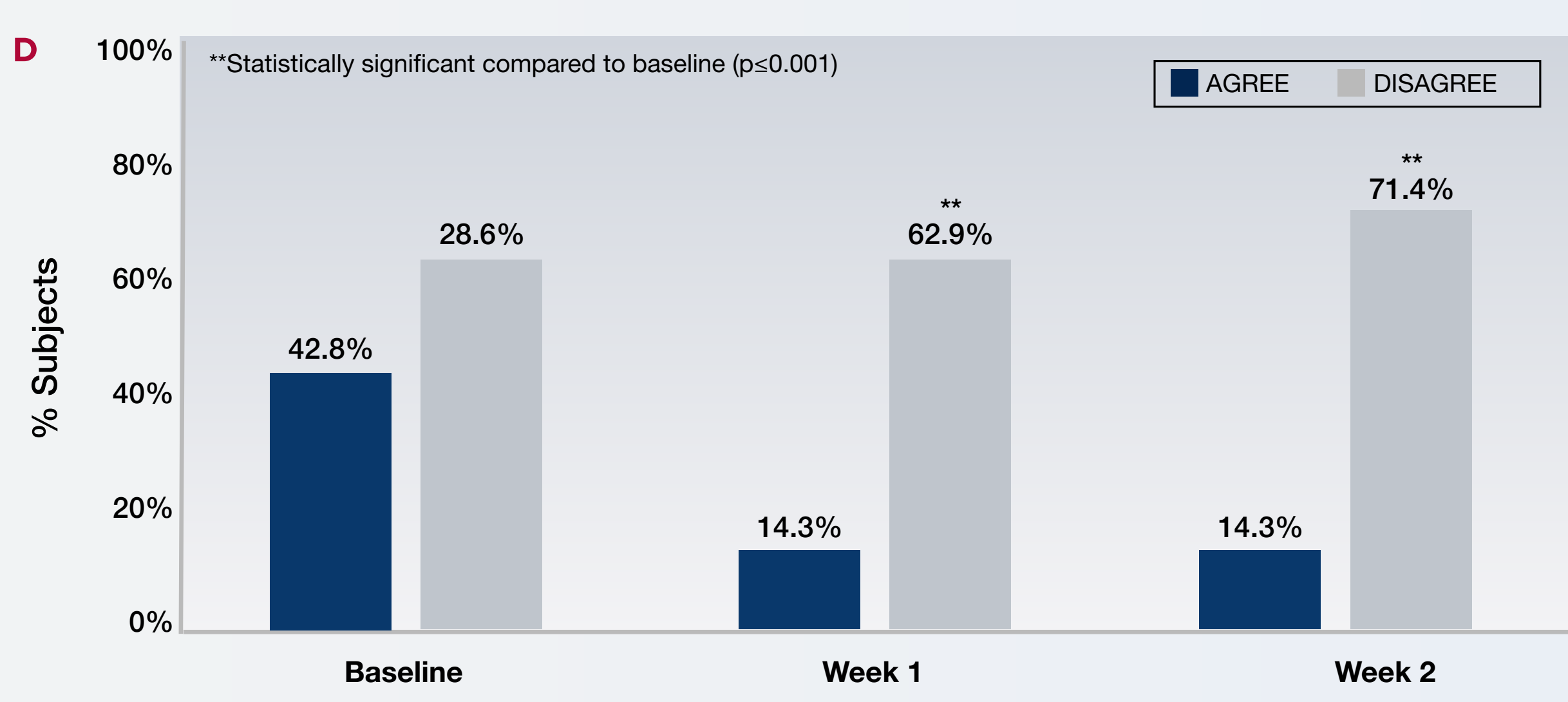

Figure 3. Skin Condition After Bathing. Self-assessments were conducted at baseline, Week 1 , and Week 2 using a subject questionnare. Subjects $(\mathrm{N}=35$ ) bathed dally utillizing the Mild Cream Body Wash as part of their bathing routine for two weeks
A. Skin irititation after bathing; B. Bkin itches after bathing; C. Skin feels dry after bathing; D. Skin appears red after bathing.

\section{CONCLUSIONS}

- Mild Cream Body Wash was well tolerated

- Statistically significant reductions in erythema, dryness, and itching were observed at Weeks 1 and 2 compared to baseline (pre-cleanser use)

- A significant decrease in irritation parameters from bathing was observed with product use

- Subjects reported the cleanser to be gentle on their eczematic skin, and gentle enough for everyday use 